\title{
THE BRÜCK CONJECTURE AND ENTIRE FUNCTIONS SHARING POLYNOMIALS WITH THEIR $k$-TH DERIVATIVES
}

\author{
FEng Lü AND HongXun Yi
}

\begin{abstract}
The purpose of this paper is twofold. The first is to establish a uniqueness theorem for entire function sharing two polynomials with its $k$-th derivative, by using the theory of normal families. Meanwhile, the theorem generalizes some related results of Rubel and Yang and of $\mathrm{Li}$ and Yi. Several examples are provided to show the conditions are necessary. The second is to generalize the Brück conjecture with the idea of sharing polynomial.
\end{abstract}

\section{Introduction and main results}

The subject on sharing values between entire functions and their derivatives was first studied by Rubel and Yang [11]. They proved a result in 1977 that if a non-constant entire function $f$ and its first derivative $f^{\prime}$ share two distinct finite numbers $a, b \mathrm{CM}$, then $f=f^{\prime}$. Since then, shared value problems, especially the case of $f$ and $f^{\prime}$ sharing two values, have been studied by many authors and a number of profound results have been obtained (see, e.g., [1, 3, 10]). To state our main result, we need the following concepts.

Let $f$ and $g$ be two non-constant meromorphic functions in the complex plane $\mathbb{C}$, and let $P$ be a polynomial or a finite complex number. If $g(z)-P(z)=0$ whenever $f(z)-P(z)=0$, we write $f(z)=P \Rightarrow g(z)=P$. Provided that $f(z)=P \Rightarrow g(z)=P$ and $g(z)=P \Rightarrow f(z)=P$, we write $f(z)=P \Leftrightarrow g(z)=$ $P$ and say that $f$ and $g$ share $P$ IM (ignoring multiplicity). If $f-P$ and $g-P$ have the same zeros with the same multiplicities, we write $f(z)=P \rightleftharpoons g(z)=$ $P$ and say that $f$ and $g$ share $P$ CM (counting multiplicity). It is assumed that the reader is familiar with the standard symbols and fundamental results of Nevanlinna theory, as found in [5, 13].

Received November 23, 2009; Revised March 18, 2010.

2010 Mathematics Subject Classification. 30D35, 30D45.

Key words and phrases. Vandermonde determinant, entire functions, Nevanlinna theory, uniqueness, normal family, differential equation.

The work was supported by the NSFC Tianyuan Mathematics Youth Fund(11026146). 
In 2006, $\mathrm{Li}$ and $\mathrm{Yi}$ [8] generalized the above result with the idea of "partially" sharing values. Here, we say that a meromorphic function $f$ "partially" shares a value $a$ with a meromorphic function $g$ if $f(z)=a \Rightarrow g(z)=a$ or $g(z)=a \Rightarrow$ $f(z)=a$. In fact, they proved the following result.

Theorem A. Let $a$ and $b$ be complex numbers such that $b \neq a, 0$, and let $f$ be a non-constant entire function. If $f(z)=a \Rightarrow f^{\prime}(z)=a$ and $f(z)=b \rightleftharpoons$ $f^{\prime}(z)=b$, then one of the following cases must occur:

(1) $f=f^{\prime}$,

(2) $f=C e^{\frac{b}{b-a} z}+a$,

where $C$ is a non-zero constant.

Recently, Lü, Xu and Chen [7] improved Theorem A with the idea of sharing polynomials. They proved the following result.

Theorem B. Let $Q_{1}(\neq 0)$ and $Q_{2}$ be two distinct polynomials, and let $f$ be a transcendental entire function. If $f(z)=Q_{1} \rightleftharpoons f^{\prime}(z)=Q_{1}$ and $f(z)=Q_{2} \Rightarrow$ $f^{\prime}(z)=Q_{2}$, then one of the following cases must occur:

(1) $f=f^{\prime}$

(2) $f(z)=Q_{2}(z)+A e^{\lambda z}$ and $(\lambda-1) Q_{1}=\lambda Q_{2}-Q_{2}^{\prime}$, where $A$ and $\lambda \neq 1$ are two non-zero constants.

It's nature to ask what will happen if the first derivative $f^{\prime}$ in Theorem $\mathrm{A}$ is replace by the general derivative $f^{(k)}$. By considering the problem, we obtain a result as follows, which is an improvement of Theorem B.

Theorem 1.1. Let $Q_{1}(\neq 0)$ and $Q_{2}$ be two distinct polynomials, let $k$ be a positive integer, and let $f$ be a transcendental entire function with all the zeros of $f-Q_{2}$ have multiplicity at least $k$. If $f(z)=Q_{1} \rightleftharpoons f^{(k)}(z)=Q_{1}$ and $f(z)=Q_{2} \Rightarrow f^{(k)}(z)=Q_{2}$, then one of the following cases must occur:

(1) $f=f^{(k)}$;

(2) $f(z)=Q_{2}(z)+A e^{\lambda z}$ and $(\mu-1) Q_{1}=\mu Q_{2}-Q_{2}^{(k)}$, where $A$ and $\lambda^{k}=$ $\mu \neq 1$ are two nonzero constants.

Remark 1.1. If $a$ is a Picard value of $f$, we say that the zeros of $f-a$ have multiplicity $\infty$. In (2) of Theorem 1.1, it's easy to see that 0 is a Picard value of $f-Q_{2}$. If we add the condition $f-Q_{2}$ has at least one zero in Theorem 1.1 , then $f$ has only one case $f=f^{(k)}$.

Remark 1.2. The hypothesis $f$ is transcendental cannot be omitted in Theorem 1.1 , as is shown by the following example.

Example 1. Let $f(z)=z^{2}, Q_{1}(z)=2 z^{2}-2 z$ and $Q_{2}=0$. Then

$$
\frac{f^{\prime}-Q_{1}}{f-Q_{1}}=2 \text { and } f=0 \Rightarrow f^{\prime}=0
$$

while it does not satisfy any case of Theorem 1.1. 
Remark 1.3. We add two examples here to point out that the case (2) in Theorem 1.1 cannot be deleted.

Example 2. Let $f(z)=A e^{\frac{z}{2}}+z, Q_{1}(z)=2-z$ and $Q_{2}(z)=z$. Then

$$
\frac{f^{\prime}-Q_{1}}{f-Q_{1}}=\frac{1}{2} \text { and } f \neq Q_{2} \text {. }
$$

Thus, it satisfies the assumption of Theorem 1.1. We have $k=1$ and $\lambda=\mu=\frac{1}{2}$.

Example 3. Let $f(z)=z+A e^{\sqrt{2} z}, Q_{1}(z)=2 z$ and $Q_{2}(z)=z$. Then

$$
\frac{f^{\prime \prime}-Q_{1}}{f-Q_{1}}=2 \text { and } f \neq Q_{2} \text {. }
$$

Thus, it satisfies the assumption of Theorem 1.1. We have $k=2, \lambda=\sqrt{2}$ and $\mu=2$.

Remark 1.4. From (1) in Theorem 1.1, we can get the forms of $f$.

Theorem 1.2. Let $f$ be a non-constant entire function and $k$ be a positive integer, let $Q$ be a polynomial, and all the zeros of $f-Q$ have multiplicity at least $k$. If $f=f^{(k)}$, then one of the following cases must occur:

(1) $k=1$ and $f(z)=A e^{z}$, where $A$ is a non-zero constant;

(2) $k=2, Q$ reduces to a constant. If $Q=0$, then $f(z)=A e^{\lambda z}$, where $\lambda^{2}=1$ and $A$ is a non-zero constant; If $Q=a$, here $a$ is a non-zero constant, then $f(z)=C_{0} e^{z}+C_{1} e^{-z}$, where $C_{0}, C_{1}$ are non-zero constants satisfying $C_{0} C_{1}=a^{2} / 4$;

(3) $k \geq 3, Q$ reduces to 0 , and $f(z)=A e^{\lambda z}$, where $\lambda^{k}=1$ and $A$ is a non-zero constant.

Remark 1.5. If $Q$ is a non-constant polynomial in Theorem 1.2, we can deduce that $k=1$ and $f=A e^{z}$, where $A$ is a non-zero constant.

Remark 1.6. The condition that all the zeros of $f-Q$ have multiplicity at least $k$ is necessary in Theorem 1.2, as is shown by the following example.

Example 4. Let $f(z)=e^{z}+e^{-z}$ and $Q=4$. When $k=2$, we have $f=f^{\prime \prime}$ and all the zeros of $f-Q$ have multiplicity at least 1 . Obviously, $C_{0} C_{1}=1 \neq$ $Q^{2} / 4=4$, thus $f$ does not satisfy any case of Theorem 1.2.

The following corollary is an immediately consequence of Theorem 1.1 and Theorem 1.2.

Corollary 1.1. Let $Q_{1}(\neq 0)$ be a polynomial and $b$ be a constant, let $f$ be $a$ transcendental entire function with all the zeros of $f-b$ have multiplicity at least $k$. If $f(z)=Q_{1} \rightleftharpoons f^{(k)}(z)=Q_{1}$ and $f(z)=b \Rightarrow f^{(k)}(z)=b$, then one of the following cases must occur:

(1) $f(z)=A e^{\lambda z}$, where $\lambda^{k}=1$ and $A$ is a non-zero constant;

(2) $k=2, b \neq 0$ and $f(z)=C_{0} e^{z}+C_{1} e^{-z}$, where $C_{0}, C_{1}$ are non-zero constants satisfying $C_{0} C_{1}=b^{2} / 4$; 
(3) $Q_{1}$ reduces to a constant, say $a$, and $f(z)=A e^{\frac{a}{a-b} z}+b$, where $A$ is a non-zero constant and $b \neq 0$.

About Theorem 1.1, the ideas of our proofs are similar as the formers in [7] and the key is to obtain the conclusion that $f$ is of finite order. For the further study of this kind of problems, we obtain a more general result, which is of independent interest.

Theorem 1.3. Let $f$ be a non-constant meromorphic function with finitely many poles, let $k$ be a positive integer, and let $\alpha=Q_{1} e^{Q}$ and $\beta=Q_{2} e^{Q}$, here $Q_{1}, Q_{2}\left(\neq Q_{1}\right)$ and $Q$ are three polynomials. If all the zeros of $f-\alpha$ have multiplicities at least $k$,

$$
f(z)=\alpha(z) \Rightarrow f^{(k)}(z)=\alpha(z) \text { and } f(z)=\beta(z) \Leftrightarrow f^{(k)}(z)=\beta(z),
$$

then $f(z)$ is of finite order.

\section{Some results of the Brück conjecture}

How is the relation between $f$ and $f^{\prime}$ if an entire function $f$ shares one value $\mathrm{CM}$ with its first derivative $f^{\prime}$ ? An important problem in this direction is the following conjecture proposed by Brück in [1].

Conjecture. Let $f$ be a non-constant entire function such that the hyper-order $\sigma_{2}(f)$ of $f$ is not a positive integer and $\sigma_{2}(f)<\infty$. If $f$ and $f^{\prime}$ share a finite value a $C M$, then

$$
\frac{f^{\prime}-a}{f-a}=c
$$

where $c$ is non-zero constant. Here, $\sigma_{2}(f)$ is defined by

$$
\sigma_{2}(f)=\varlimsup_{r \rightarrow \infty} \frac{\log \log T(r, f)}{\log r} .
$$

The conjecture for the case $a=0$ had been proved by R. Brück in [1]. From differential equations

$$
\frac{f^{\prime}-a}{f-a}=e^{z^{n}}, \frac{f^{\prime}-a}{f-a}=e^{e^{z}}
$$

we see that when the hyper-order $\sigma_{2}(f)$ is a positive integer or infinite, the conjecture does not hold.

The conjecture for the case that $f$ is of finite order had been proved by Gundersen and Yang [3], the case that $f$ is of infinite order with $\sigma_{2}(f)<\frac{1}{2}$ had been proved by Chen and Shon [2]. But the case $\sigma_{2}(f) \geq \frac{1}{2}$ is still open.

Under some additional assumptions, there are many results related to the Brück conjecture, see, e.g., [12, Theorem 1], [17, Theorem 1] and [18, Theorem $1.1]$.

It is interesting to ask what happens if $f$ is replaced by $f^{n}$ in the Brück conjecture. From (2.1), we see that the conjecture does not hold when $n=1$. Thus, we only need to discuss the problem when $n \geq 2$. Recently, Yang and 
Zhang [14] proved that the Brück conjecture holds for the function $f^{n}$, and the order restriction on $f$ does not needed if $n$ is relatively large. Actually, they proved the following result.

Theorem B. Let $f$ be a non-constant entire function, $n \geq 7$ be an integer, and let $F=f^{n}$. If $F$ and $F^{\prime}$ share $1 C M$, then $F=F^{\prime}$ and $f$ assumes the form

$$
f(z)=c e^{\frac{1}{n} z}
$$

where $c$ is a non-zero constant.

Very recently, in Zhang's doctoral dissertation [16] he obtained a general result.

Theorem C. Let $f$ be a non-constant entire function, let $n$ and $k$ be two integers with $n \geq k+1$, and let $F=f^{n}$. If $F$ and $F^{(k)}$ share $1 C M$, then $F=F^{(k)}$ and $f$ assumes the form

$$
f(z)=c e^{\frac{\lambda}{n} z}
$$

where $c$ is a non-zero constant and $\lambda^{k}=1$.

In the following, we continue to study the class of special functions $F=f^{n}$, and replace the constant $a$ by a polynomial $Q$. We have the next conclusion.

Theorem 2.1. Let $f$ be a transcendental entire function, let $n$ and $k$ be two integers with $n \geq k+1$, and let $F=f^{n}$ and $Q \neq 0$ be a polynomial. If $F$ and $F^{(k)}$ share $Q C M$, then $F=F^{(k)}$ and $f$ assumes the form

$$
f(z)=c e^{\frac{\lambda}{n} z},
$$

where $c$ is a non-zero constant and $\lambda^{k}=1$.

Remark 2.1. Obviously, all the zeros of $F$ have multiplicity at least $k+1$ in Theorem 2.1. Thus, by Corollary 1.1, it's easy to deduce Theorem 2.1.

Remark 2.2. We give an example to show the hypothesis that $f$ is transcendental is necessary in Theorem 2.1 .

Let $f(z)=z$ and $Q(z)=2 z^{2}-2 z$, and let $n=2, k=1$. Then $F(z)=$ $f^{2}(z)=z^{2}$ and

$$
\frac{F^{\prime}-Q}{F-Q}=2
$$

but $F \neq F^{\prime}$

Remark 2.3. In Theorem 2.1, if $Q$ is a constant, the condition that $f$ is transcendental can be omitted. Thus, for the special case $F=f^{n}$, the Brück conjecture is true. Meanwhile, Theorem 2.1 improves Theorem B and Theorem C.

Remark 2.4. It's easy to deduce that the conjecture holds for the class of special functions $F=f^{n}$ with $n \geq 2$. 
Theorem 2.2. Let $f$ be a non-constant entire function, let $n$ and $k$ be two integers with $n \geq k+1$, and let $F=f^{n}$ and $Q \neq 0$ be a polynomial. If $F$ and $F^{(k)}$ share $Q C M$, then

$$
\frac{F^{(k)}-Q}{F-Q}=c
$$

where $c$ is a non-zero constant.

Remark 2.5. In fact, by Theorem 2.1, we need only to prove Theorem 2.2 holds when $f$ is a polynomial. Obviously, it holds.

\section{Some lemmas}

In order to prove our theorems, we need the following lemmas.

Lemma 3.1 ([7]). Let $\left\{f_{n}\right\}$ be a family of functions meromorphic (analytic) on the unit disc $\triangle$. If $a_{n} \rightarrow a,|a|<1$, and $f_{n}^{\sharp}\left(a_{n}\right) \rightarrow \infty$, then there exist

(a) a subsequence of $f_{n}$ (which we still write as $\left.\left\{f_{n}\right\}\right)$;

(b) points $z_{n} \rightarrow z_{0}\left|z_{0}\right|<1$;

(c) positive numbers $\rho_{n} \rightarrow 0$

such that $f_{n}\left(z_{n}+\rho_{n} \xi\right)=g_{n}(\xi) \rightarrow g(\xi)$ locally uniformly, where $g$ is a nonconstant meromorphic (entire) function on $\mathbb{C}$, such that

$$
\rho_{n} \leq \frac{M}{f_{n}^{\sharp}\left(a_{n}\right)},
$$

where $M$ is a constant which is independent of $n$.

Here, as usual, $g^{\sharp}(\xi)=\frac{\left|g^{\prime}(\xi)\right|}{1+|g(\xi)|^{2}}$ is the spherical derivative.

Lemma 3.2 ([6]). Let $f$ be a meromorphic function of infinite order on $\mathbb{C}$. Then there exist points $z_{n} \rightarrow \infty$, such that for every $N>0, f^{\sharp}\left(z_{n}\right)>\left|z_{n}\right|^{N}$ if $n$ is sufficiently large.

Lemma 3.3 ([9, Corollary 1.4]). Let $f$ be a non-constant entire function of hyper-order $\sigma_{2}(f)<1$, and let $Q \neq 0$ and $P$ be two polynomials. If $f$ is a solution of the differential equation

$$
\frac{f^{(k)}-Q}{f-Q}=e^{P},
$$

then $P$ must be a constant.

\section{Proof of Theorem 1.3}

In the following, we will prove the theorem, and some ideas of the result are based on [4].

Let $H=f-\alpha$. Then we have that all the zeros of $H$ have multiplicities at least $k$ and

$$
H=0 \Rightarrow H^{(k)}=\alpha-\alpha^{(k)}, H=\beta-\alpha \Leftrightarrow H^{(k)}=\beta-\alpha^{(k)},
$$


here $\alpha^{(k)}=P_{1} e^{Q}$ and $P_{1}$ is a polynomial. Let

$$
\gamma=\beta-\alpha=\left(Q_{2}-Q_{1}\right) e^{Q}=P e^{Q}
$$

and

$$
\theta=\beta-\alpha^{(k)}=\left(Q_{2}-P_{1}\right) e^{Q}=P_{2} e^{Q} .
$$

Define $F=\frac{H}{\gamma}$. We now proceed the proof by distinguishing two cases.

Case 1. $F$ is of finite order.

Hence $f=F \gamma+\alpha$ is of finite order as well.

Case 2. $F$ is of infinite order.

By Lemma 3.2, there exist $w_{n} \rightarrow \infty$, such that for every $N>0$, if $n$ is sufficiently large

$$
F^{\sharp}\left(w_{n}\right)>\left|w_{n}\right|^{N} \text {. }
$$

First we structure a family of holomorphic functions.

Since $f$ has only finitely many poles, then $H=f-\alpha$ has only finitely many poles. Obviously, $\gamma=P e^{Q}$ has only finitely many zeros. So there exists a $r>0$ such that $H(z)$ is analytic and $\gamma(z) \neq 0$ in $D=\{z:|z| \geq r\}$. It is easy to obtain that $F(z)=\frac{H(z)}{\gamma(z)}$ is analytic in $D$. In view of $w_{n} \rightarrow \infty$ as $n \rightarrow \infty$, without loss of generality, we may assume $\left|w_{n}\right| \geq r+1$ for all $n$. Define $D_{1}=\{z:|z|<1\}$ and

$$
F_{n}(z)=F\left(w_{n}+z\right)=\frac{H\left(w_{n}+z\right)}{\gamma\left(w_{n}+z\right)} .
$$

Noting that $\left|w_{n}\right| \geq r+1$ for all $n$, then we have, for each $z \in D_{1}$,

$$
\left|w_{n}+z\right| \geq\left|w_{n}\right|-|z| \geq r
$$

which implies that $w_{n}+z \in D$ for each $z \in D_{1}$. Observing that $F(z)$ is analytic in $D$, so $F_{n}(z)=F\left(w_{n}+z\right)$ is analytic in $D_{1}$. Therefore, all $F_{n}(z)$ are analytic in $D_{1}$. Thus, we have structured a family $\left(F_{n}\right)_{n}$ of holomorphic functions.

In what follows, we prove that $\left(F_{n}\right)_{n}$ is normal at $z=0$, here

$$
F_{n}(z)=F\left(w_{n}+z\right)=\frac{H\left(w_{n}+z\right)}{\gamma\left(w_{n}+z\right)} .
$$

Otherwise, we can apply Lemma 3.1. Choosing an appropriate subsequence of $\left(F_{n}\right)_{n}$ if necessary, we may assume that there exist sequence $\left(z_{n}\right)_{n} \in D_{1}$ and $\left(\rho_{n}\right)_{n}$ such that $\left|z_{n}\right|<r<1, \rho_{n} \rightarrow 0$ and

$$
g_{n}(\zeta)=F_{n}\left(z_{n}+\rho_{n} \zeta\right)=\frac{H\left(w_{n}+z_{n}+\rho_{n} \zeta\right)}{\gamma\left(w_{n}+z_{n}+\rho_{n} \zeta\right)} \rightarrow g(\zeta)
$$

locally uniformly in $\mathbb{C}$, where $g$ is a non-constant entire function, whose zeros have multiplicity at least $k$ and

$$
\rho_{n} \leq \frac{M}{F_{n}^{\sharp}(0)}=\frac{M}{F^{\sharp}\left(w_{n}\right)}
$$


for a positive number $M$. From (4.1) and (4.3), we deduce that, for every $N>0$, if $n$ is sufficiently large,

$$
\rho_{n} \leq M\left|w_{n}\right|^{-N}
$$

We claim that

$$
\rho_{n}^{k} \frac{H^{(k)}\left(w_{n}+z_{n}+\rho_{n} \zeta\right)}{\gamma\left(w_{n}+z_{n}+\rho_{n} \zeta\right)} \rightarrow g^{(k)}(\zeta) .
$$

We will use the mathematical induction to prove the claim. By (4.2), the claim is right when $k=0$. We assume that the claim is right when $k=l$, that is

$$
\rho_{n}^{l} \frac{H^{(l)}\left(w_{n}+z_{n}+\rho_{n} \zeta\right)}{\gamma\left(w_{n}+z_{n}+\rho_{n} \zeta\right)} \rightarrow g^{(l)}(\zeta)
$$

Now we prove the claim is also right when $k=l+1$. Let

$$
G_{n}(\zeta)=\rho_{n}^{l} \frac{H^{(l)}\left(w_{n}+z_{n}+\rho_{n} \zeta\right)}{\gamma\left(w_{n}+z_{n}+\rho_{n} \zeta\right)}
$$

then

$$
\begin{aligned}
& G_{n}^{\prime}(\zeta) \\
= & \rho_{n}^{l+1} \frac{H^{(l+1)}\left(w_{n}+z_{n}+\rho_{n} \zeta\right)}{\gamma\left(w_{n}+z_{n}+\rho_{n} \zeta\right)}-\rho_{n}^{l+1} \frac{H^{(l)}\left(w_{n}+z_{n}+\rho_{n} \zeta\right) \gamma^{\prime}\left(w_{n}+z_{n}+\rho_{n} \zeta\right)}{\gamma\left(w_{n}+z_{n}+\rho_{n} \zeta\right)^{2}} \\
= & \rho_{n}^{l+1} \frac{H^{(l+1)}\left(w_{n}+z_{n}+\rho_{n} \zeta\right)}{\gamma\left(w_{n}+z_{n}+\rho_{n} \zeta\right)}-\rho_{n} \frac{G_{n}(\zeta) \gamma^{\prime}\left(w_{n}+z_{n}+\rho_{n} \zeta\right)}{\gamma\left(w_{n}+z_{n}+\rho_{n} \zeta\right)} \rightarrow g^{(l+1)}(\zeta) .
\end{aligned}
$$

Note that (4.4), we deduce

$$
\rho_{n} \frac{G_{n}(\zeta) \gamma^{\prime}\left(w_{n}+z_{n}+\rho_{n} \zeta\right)}{\gamma\left(w_{n}+z_{n}+\rho_{n} \zeta\right)}=\rho_{n} \frac{G_{n}(\zeta)\left(P^{\prime}+P Q^{\prime}\right)\left(w_{n}+z_{n}+\rho_{n} \zeta\right)}{P\left(w_{n}+z_{n}+\rho_{n} \zeta\right)} \rightarrow 0 .
$$

Then

$$
\rho_{n}^{l+1} \frac{H^{(l+1)}\left(w_{n}+z_{n}+\rho_{n} \zeta\right)}{\gamma\left(w_{n}+z_{n}+\rho_{n} \zeta\right)} \rightarrow g^{(l+1)}(\zeta)
$$

So we complete the proof of the claim.

Obviously, $g^{(k)} \neq 0$, for otherwise $g$ would be a polynomial of degree less than $k$, and so could not have zeros of multiplicity at least $k$. In the following, we will prove

(1) $g=0 \Rightarrow g^{(k)}=0$,

(2) $g=1 \Leftrightarrow g^{(k)}=0$.

Firstly, we prove (1). Suppose that $g\left(\zeta_{0}\right)=0$, then by Hurwitz's theorem, there exists a sequence $\left(\zeta_{n}\right)_{n}, \zeta_{n} \rightarrow \zeta_{0}$ such that (for $n$ sufficiently large)

$$
g_{n}\left(\zeta_{n}\right)=\frac{H\left(w_{n}+z_{n}+\rho_{n} \zeta_{n}\right)}{\gamma\left(w_{n}+z_{n}+\rho_{n} \zeta_{n}\right)}=0 .
$$

Thus $H\left(w_{n}+z_{n}+\rho_{n} \zeta_{n}\right)=0$ and

$$
H^{(k)}\left(w_{n}+z_{n}+\rho_{n} \zeta_{n}\right)=\left(Q_{1}-P_{1}\right) e^{Q}\left(w_{n}+z_{n}+\rho_{n} \zeta_{n}\right) .
$$


By (4.4) and (4.5) we derive that

$$
\begin{aligned}
g^{(k)}\left(\zeta_{0}\right) & =\lim _{n \rightarrow \infty} \rho_{n}^{k} \frac{H^{(k)}\left(w_{n}+z_{n}+\rho_{n} \zeta_{n}\right)}{\gamma\left(w_{n}+z_{n}+\rho_{n} \zeta_{n}\right)} \\
& =\lim _{n \rightarrow \infty} \rho_{n}^{k} \frac{\left(Q_{1}-P_{1}\right)\left(w_{n}+z_{n}+\rho_{n} \zeta_{n}\right)}{P\left(w_{n}+z_{n}+\rho_{n} \zeta_{n}\right)}=0 .
\end{aligned}
$$

Thus $g(\zeta)=0 \Rightarrow g^{(k)}(\zeta)=0$. It's (1).

Similarly, we can prove $g=1 \Rightarrow g^{(k)}=0$. Now, we prove

$$
g^{(k)}=0 \Rightarrow g=1 \text {. }
$$

From (4.4) and (4.5), we have

$$
\rho_{n}^{k} \frac{H^{(k)}\left(w_{n}+z_{n}+\rho_{n} \zeta\right)-\theta\left(w_{n}+z_{n}+\rho_{n} \zeta\right)}{\gamma\left(w_{n}+z_{n}+\rho_{n} \zeta\right)} \rightarrow g^{(k)}(\zeta) .
$$

Suppose that $g^{(k)}\left(\eta_{0}\right)=0$, we know $g^{(k)} \neq 0$. Hence by (4.6) and Hurwitz's theorem, there exists a sequence $\left(\eta_{n}\right)_{n}, \eta_{n} \rightarrow \eta_{0}$ such that (for $n$ sufficiently large)

$$
H^{(k)}\left(w_{n}+z_{n}+\rho_{n} \eta_{n}\right)=\theta\left(w_{n}+z_{n}+\rho_{n} \eta_{n}\right) .
$$

By the assumption, we get $H\left(w_{n}+z_{n}+\rho_{n} \eta_{n}\right)=\gamma\left(w_{n}+z_{n}+\rho_{n} \eta_{n}\right)$.

Then

$$
g\left(\eta_{0}\right)=\lim _{n \rightarrow \infty} \frac{H\left(w_{n}+z_{n}+\rho_{n} \eta_{n}\right)}{\gamma\left(w_{n}+z_{n}+\rho_{n} \eta_{n}\right)}=1 .
$$

Thus, we prove $g^{(k)}=0 \Rightarrow g=1$ and (2).

From (1) and (2), it's easy to deduce that $g \neq 0$. Thus, we set $g=A e^{\lambda z}$. But it contradicts with $g=1 \Rightarrow g^{(k)}=0$.

All the foregoing discussion shows that $\left(F_{n}\right)_{n}$ is normal at $z=0$.

On the other hand, it follows from the fact $F_{n}^{\sharp}(0)=F^{\sharp}\left(w_{n}\right) \rightarrow \infty$ as $n \rightarrow \infty$ and Marty's criterion that $\left(F_{n}\right)_{n}$ is not normal at $z=0$, a contradiction. Hence, Case 2 cannot occur.

This completes the proof of Theorem 1.3.

\section{Proof of Theorem 1.1}

From Theorem 1.3, we know that $f$ is of finite order. Thus, the hyper-order $\sigma_{2}(f)=0$. Then, by Lemma 3.3, we have

$$
\mu=\frac{f^{(k)}-Q_{1}}{f-Q_{1}},
$$

where $\mu$ is a non-zero constant. Rewrite it as

$$
f^{(k)}=\mu f+(1-\mu) Q_{1} .
$$

If $\mu=1$, we obtain $f=f^{(k)}$, which is (1). Now, we assume that $\mu \neq 1$. Let $z_{0}$ be a zero of $f-Q_{2}$, by the assumption we have $f^{(k)}\left(z_{0}\right)=Q_{2}\left(z_{0}\right)$. Put $z_{0}$ into 
(5.2), we have

$$
Q_{2}\left(z_{0}\right)-Q_{1}\left(z_{0}\right)=0 .
$$

Note that $Q_{2}-Q_{1} \neq 0$, we deduce that all the zeros of $f-Q_{2}$ are the zeros of $Q_{2}-Q_{1}$ and $f-Q_{2}$ has only finitely many zeros. Set $f=Q_{2}+P_{1} e^{Q}$, here $P_{1}$ is a non-zero polynomial and $Q$ is a non-constant polynomial. Then $f^{(k)}=$ $Q_{2}^{(k)}+\left(P_{1} Q^{\prime k}+P_{2}\right) e^{Q}$ and $P_{2}$ is a polynomial with $\operatorname{deg}\left(P_{2}\right)<\operatorname{deg}\left(P_{1} Q^{\prime k}\right)$.

Substitute them into (5.2), we deduce that

$$
Q_{2}^{(k)}+\left(P_{1} Q^{\prime k}+P_{2}\right) e^{Q}=\mu P_{1} e^{Q}+\mu Q_{2}+(1-\mu) Q_{1} .
$$

Then, we get

$$
Q_{2}^{(k)}=\mu Q_{2}+(1-\mu) Q_{1}
$$

and

$$
P_{1} Q^{\prime k}+P_{2}=\mu P_{1}
$$

From (5.6), we get $Q^{\prime k}=\mu$ and $P_{2}=0$. Thus, $Q^{\prime}$ is a constant, say $Q^{\prime}=\lambda$. Then $Q(z)=\lambda z+b$ and $\mu=\lambda^{k}$, here $b$ is a constant. So we obtain $f(z)=$ $Q_{2}(z)+P_{1}(z) e^{\lambda z+b}, f^{(k)}=Q_{2}^{(k)}+\left(P_{1} \lambda^{k}+P_{2}\right) e^{Q}$. It's easy to deduce that $\operatorname{deg}\left(P_{2}\right)=\operatorname{deg}\left(P_{1}^{\prime}\right)$. From $P_{2}=0$, we get $P_{1}$ is a constant. Let $P_{1} e^{b}=A$. Then we have $(2)$.

Hence, we complete the proof of this theorem.

\section{Proof of Theorem 1.2}

The fact $f=f^{(k)}$ implies that $f$ is of finite order 1. Firstly, we assume that $Q=0$. From the assumptions $f=f^{(k)}$ and all the zeros of $f-Q$ have multiplicity at least $k$, it's easy to deduce that 0 is a Picard value of $f$. Set $f(z)=A e^{\lambda z}, A$ is a non-zero constant. Then, $f=f^{(k)}$ yields $\lambda^{k}=1$.

Suppose that $k=1$, then, it's easy to get $f(z)=A e^{z}$.

In the following, we assume that $Q \neq 0$ and $k \geq 2$. By solving the differential equation $f=f^{(k)}$, we have

$$
f(z)=\sum_{j=0}^{k-1} C_{j} \exp ^{\omega^{j} z},
$$

where $\omega=\exp ^{2 \pi i / k}$ and $C_{j}$ are constants. Noting that $f$ is non-constant, then there exist $C_{j} \in\left\{C_{0}, C_{1}, \ldots, C_{k-1}\right\}$ such that $C_{j} \neq 0$. Denote the non-zero constants in $\left\{C_{j}\right\}$ by $C_{j_{m}} 0 \leq j_{m} \leq k-1$ and $m=0,1, \ldots, s, s \leq k-1$. Thus, rewriting $(6.1)$ as

$$
f(z)=\sum_{m=0}^{s} C_{j_{m}} \exp ^{\omega^{j_{m}} z} .
$$


Differentiating (6.2) yields

$$
f^{(t)}(z)=\sum_{m=0}^{s} C_{j_{m}}\left(\omega^{j_{m}}\right)^{t} \exp ^{\omega^{j_{m}} z},(t=1,2 \ldots, k-1) .
$$

Suppose that $f-Q$ has finitely many zeros, then we can set $f=Q+P e^{\lambda z}$. By differentiating it $k$ times, we deduce

$$
f^{(k)}=Q^{(k)}+\left[\lambda^{k} P+\lambda^{k-1} P^{\prime}+H\left(P^{\prime \prime}, P^{\prime \prime \prime}, \ldots, P^{(k)}\right)\right] e^{\lambda z},
$$

where $H\left(P^{\prime \prime}, P^{\prime \prime \prime}, \ldots, P^{(k)}\right)$ is the linear combination of $P^{\prime \prime}, P^{\prime \prime \prime}, \ldots, P^{(k)}$. Substituting the above forms of $f$ and $f^{(k)}$ into $f=f^{(k)}$, we derive that

$$
Q=Q^{(k)}, P=\lambda^{k} P+\lambda^{k-1} P^{\prime}+H\left(P^{\prime}, P^{\prime \prime}, \ldots, P^{(k)}\right),
$$

which implies that $Q=0$ or $Q$ is transcendental, a contradiction.

Thus, in what follows, we assume that $f-Q$ has infinitely many zeros $z_{n}=r_{n} e^{\theta_{n}}$, where $0 \leq \theta_{n}<2 \pi$. Without loss of generality, we may assume that $\theta_{n} \rightarrow \theta_{0}$ and $r_{n} \rightarrow \infty$ as $n \rightarrow \infty$. Substituting $z_{n}$ into (6.2) and (6.3), we have

$$
f\left(z_{n}\right)=\sum_{m=0}^{s} C_{j_{m}} \exp ^{\omega^{j_{m}} z_{n}}=Q\left(z_{n}\right)
$$

and

$$
f^{(t)}\left(z_{n}\right)=\sum_{m=0}^{s} C_{j_{m}}\left(\omega^{j_{m}}\right)^{t} \exp ^{\omega^{j_{m}} z_{n}}=Q^{(t)}\left(z_{n}\right),(t=1,2 \ldots, k-1) .
$$

Next, we consider into two cases.

Case 1. $s=k-1$.

From (6.4) and (6.5), we have

$$
\left(\begin{array}{c}
Q\left(z_{n}\right) \\
Q^{\prime}\left(z_{n}\right) \\
\vdots \\
Q^{(k-1)}\left(z_{n}\right)
\end{array}\right)=\left(\begin{array}{cccc}
C_{j_{0}} & C_{j_{1}} & \cdots & C_{j_{k-1}} \\
C_{j_{0}} \omega^{j_{0}} & C_{j_{1}} \omega^{j_{1}} & \cdots & C_{j_{k-1}} \omega^{j_{k-1}} \\
\vdots & & & \\
C_{j_{0}}\left(\omega^{j_{0}}\right)^{k-1} & C_{j_{1}}\left(\omega^{j_{1}}\right)^{k-1} & \cdots & C_{j_{k-1}}\left(\omega^{j_{k-1}}\right)^{k-1}
\end{array}\right)\left(\begin{array}{c}
\exp ^{\omega^{j_{0}} z_{n}} \\
\exp ^{\omega^{j_{1}} z_{n}} \\
\vdots \\
\exp ^{\omega^{j_{k-1}} z_{n}}
\end{array}\right) .
$$

We know

$$
\begin{array}{r}
\operatorname{det}\left(\begin{array}{cccc}
C_{j_{0}} & C_{j_{1}} & \cdots & C_{j_{k-1}} \\
C_{j_{0}} \omega^{j_{0}} & C_{j_{1}} \omega^{j_{1}} & \cdots & C_{j_{k-1}} \omega^{j_{k-1}} \\
\vdots & & & \\
C_{j_{0}}\left(\omega^{j_{0}}\right)^{k-1} & C_{j_{1}}\left(\omega^{j_{1}}\right)^{k-1} & \cdots & C_{j_{k-1}}\left(\omega^{j_{k-1}}\right)^{k-1}
\end{array}\right) \\
=C_{j_{0}} C_{j_{1}} \cdots C_{j_{k-1}} \operatorname{det}\left(\begin{array}{cccc}
1 & 1 & \cdots & 1 \\
\omega^{j_{0}} & \omega^{j_{1}} & \cdots & \omega^{j_{k-1}} \\
\vdots & & & \\
\left(\omega^{j_{0}}\right)^{k-1} & \left(\omega^{j_{1}}\right)^{k-1} & \cdots & \left(\omega^{j_{k-1}}\right)^{k-1}
\end{array}\right)
\end{array}
$$




$$
=C_{j_{0}} C_{j_{1}} \cdots C_{j_{k-1}} \prod_{0 \leq q<p \leq k-1}\left(\omega^{j_{p}}-\omega^{j_{q}}\right) .
$$

It is a Vandermonde determinant.

Noting that $\omega^{j_{p}} \neq \omega^{j_{q}}(0 \leq q<p \leq k-1)$, we obtain that the system of linear equations of $\exp ^{\omega^{j} z_{n}}$, $\exp ^{\omega^{j_{1}} z_{n}}, \ldots, \exp ^{\omega^{j_{k-1}} z_{n}}$ has a unique solution. A routine calculation leads to the solution that

$$
\exp ^{\omega^{j_{p}} z_{n}}=L_{p}\left(Q, Q^{\prime}, \ldots, Q^{(k-1)}\right)\left(z_{n}\right),(0 \leq p \leq k-1) .
$$

where $L_{p}\left(Q, Q^{\prime}, \ldots, Q^{(k-1)}\right) \neq 0$ denotes the linear combination of $Q, Q^{\prime}, \ldots$, $Q^{(k-1)}$, which may be different as $p$ changes.

Now, we discuss the solution. Obviously,

$\left|L_{p}\left(Q, Q^{\prime}, \ldots, Q^{(k-1)}\right)\left(z_{n}\right)\right|=\left|\exp ^{\omega^{j} z_{n}}\right|=\exp ^{r_{n} \cos \left(\theta_{n}+\frac{2 p \pi}{k}\right)},(0 \leq p \leq k-1)$.

It is clear that $L_{p}\left(Q, Q^{\prime}, \ldots, Q^{(k-1)}\right)$ is a polynomial. Thus, as $n \rightarrow \infty$, by (6.7) we can deduce that

$$
\cos \left(\theta_{0}+\frac{2 p \pi}{k}\right)=0,(0 \leq p \leq k-1) .
$$

Otherwise, we have $\cos \left(\theta_{0}+\frac{2 p \pi}{k}\right)>0$ or $\cos \left(\theta_{0}+\frac{2 p \pi}{k}\right)<0$.

If $\cos \left(\theta_{0}+\frac{2 p \pi}{k}\right)>0$, then we can assume (for $n$ large enough) $\cos \left(\theta_{n}+\frac{2 p \pi}{k}\right)>$ $\delta$, here $\delta$ is a small positive number. Thus, as $n \rightarrow \infty$, by (6.7) we have

$$
1=\frac{\exp ^{r_{n} \cos \left(\theta_{n}+\frac{2 p \pi}{k}\right)}}{\left|L_{p}\left(Q, Q^{\prime}, \ldots, Q^{(k-1)}\right)\left(z_{n}\right)\right|}>\frac{\exp ^{r_{n} \delta}}{\left|L_{p}\left(Q, Q^{\prime}, \ldots, Q^{(k-1)}\right)\left(z_{n}\right)\right|} \rightarrow \infty
$$

a contradiction.

If $\cos \left(\theta_{0}+\frac{2 p \pi}{k}\right)<0$, then we can assume (for $n$ large enough) $\cos \left(\theta_{n}+\frac{2 p \pi}{k}\right)<$ $-\delta$, here $\delta$ is a small positive number. Thus, as $n \rightarrow \infty$, by $(6.7)$ we have

$$
1=\frac{\exp ^{r_{n} \cos \left(\theta_{n}+\frac{2 p \pi}{k}\right)}}{\left|L_{p}\left(Q, Q^{\prime}, \ldots, Q^{(k-1)}\right)\left(z_{n}\right)\right|}<\frac{\exp ^{-r_{n} \delta}}{\left|L_{p}\left(Q, Q^{\prime}, \ldots, Q^{(k-1)}\right)\left(z_{n}\right)\right|} \rightarrow 0,
$$

a contradiction.

Observing that $0 \leq p, q \leq k-1$, by (6.8), we deduce

$$
\left|\frac{2 p \pi}{k}-\frac{2 q \pi}{k}\right|=\pi,(0 \leq p \neq q \leq k-1) .
$$

Let $p=0$ and $q=k-1$. Substitute them into (6.9), we have

$$
2(k-1)=k,
$$

that is $k=2$. Thus, $k$ must be 2 .

Now we discuss the equation $f=f^{(k)}$ again when $k=2$. From the above discussion, we can obtain $\omega_{0}=1, \omega_{1}=-1$ and

$$
C_{0} e^{z_{n}}=\frac{Q\left(z_{n}\right)+Q^{\prime}\left(z_{n}\right)}{2}, C_{1} e^{-z_{n}}=\frac{Q\left(z_{n}\right)-Q^{\prime}\left(z_{n}\right)}{2} .
$$


Thus,

$$
C_{0} C_{1}=\frac{\left(Q\left(z_{n}\right)+Q^{\prime}\left(z_{n}\right)\right)\left(Q\left(z_{n}\right)-Q^{\prime}\left(z_{n}\right)\right)}{4} .
$$

If $C_{0} C_{1}=\frac{\left(Q+Q^{\prime}\right)\left(Q-Q^{\prime}\right)}{4}$, it is obvious that $Q$ deduce to a constant. Set $Q=a$, then $C_{0} C_{1}=\frac{a^{2}}{4}$.

If $C_{0} C_{1} \neq \frac{\left(Q+Q^{\prime}\right)\left(Q-Q^{\prime}\right)}{4}$, then it is not difficult to derive that $f-Q$ has only finitely many zeros, a contradiction.

Case 2. $s<k-1$.

Then, by $(6.5)$, we can choose $t=1,2, \ldots, s+1$. Then they form a system of linearly equation of $\exp ^{\omega^{j_{0}} z_{n}}, \exp ^{\omega^{j_{1}} z_{n}}, \ldots, \exp ^{\omega^{j_{s}} z_{n}}$.

Similarly as Case 1, we deduce

$$
\exp ^{\omega^{j} z_{n}}=L_{p}\left(Q^{\prime}, Q^{\prime \prime}, \ldots, Q^{(m)}\right)\left(z_{n}\right),(0 \leq p \leq s) .
$$

where $L_{p}\left(Q^{\prime}, Q^{\prime \prime}, \ldots, Q_{(m)}\right)$ denotes the linear combination of $Q^{\prime}, Q^{\prime \prime}, \ldots, Q^{(m)}$, which may be different as $p$ changes. Substituting them into (6.4), we have

$$
L\left(Q^{\prime}, Q^{\prime \prime}, \ldots, Q^{(m)}\right)\left(z_{n}\right)=Q\left(z_{n}\right),
$$

here $L\left(Q^{\prime}, Q^{\prime \prime}, \ldots, Q^{(m)}\right)$ denotes the linear combination of $Q^{\prime}, Q^{\prime \prime}, \ldots, Q^{(m)}$. Noting that $L\left(Q^{\prime}, Q^{\prime \prime}, \ldots, Q^{(m)}\right) \neq Q$, we deduce that $f-Q$ has only finitely many zeros, a contradiction.

Hence, we complete the proof of this theorem.

Acknowledgment. The authors are grateful to the referee for his(or her) valuable suggestions and comments.

\section{References}

[1] R. Brück, On entire functions which share one value CM with their first derivative, Results Math. 30 (1996), no. 1-2, 21-24.

[2] Z. X. Chen and K. H. Shon, On conjecture of R. Brück concerning the entire function sharing one value CM with its derivative, Taiwanese J. Math. 8 (2004), no. 2, 235-244.

[3] G. G. Gundersen and L. Z. Yang, Entire functions that share one value with one or two of their derivatives, J. Math. Anal. Appl. 223 (1998), no. 1, 88-95.

[4] J. Grahl and C. Meng, Entire functions sharing a polynomial with their derivatives and normal families, Analysis (Munich) 28 (2008), no. 1, 51-61.

[5] W. Hayman, Meromorphic Functions, Clarendon Press, Oxford, 1964.

[6] X. J. Liu, S. Nevo, and X. C. Pang, On the kth derivative of meromorphic functions with zeros of multiplicity at least $k+1$, J. Math. Anal. Appl. 348 (2008), no. 1, 516-529.

[7] F. Lü, J. F. Xu, and A. Chen, Entire functions sharing polynomials with their first derivatives, Arch. Math. (Basel) 92 (2009), no. 6, 593-601.

[8] J. T. Li and H. X. Yi, Normal families and uniqueness of entire functions and their derivatives, Arch. Math. (Basel) 87 (2006), no. 1, 52-59.

[9] X. M. Li and C. C. Gao, Entire functions sharing one polynomial with their derivatives, Proc. Indian Acad. Sci. Math. Sci. 118 (2008), no. 1, 13-26.

[10] E. Mues and N. Steinmetz, Meromorphe Funktionen, die mit ihrer Ableitung Werte teilen, Manuscripta Math. 29 (1979), no. 2-4, 195-206. 
[11] L. A. Rubel and C. C. Yang, Values shared by an entire function and its derivative, Complex analysis (Proc. Conf., Univ. Kentucky, Lexington, Ky., 1976), pp. 101-103. Lecture Notes in Math., Vol. 599, Springer, Berlin, 1977.

[12] J. Wang and H. X. Yi, The uniqueness of entire functions that share a small function with its differential polynomials, Indian J. Pure Appl. Math. 35 (2004), no. 9, 1119-1129.

[13] C. C. Yang and H. X. Yi, Uniqueness Theory of Meromorphic Functions, Mathematics and its Applications, 557. Kluwer Academic Publishers Group, Dordrecht, 2003.

[14] L. Z. Yang and J. L. Zhang, Non-existence of meromorphic solutions of a Fermat type functional equation, Aequationes Math. 76 (2008), no. 1-2, 140-150.

[15] L. Zalcman, A heuristic principle in complex function theory, Amer. Math. Monthly 82 (1975), no. 8, 813-817.

[16] J. L. Zhang, Researches on Brück Conjecture and Fermat Diophantine Equations over function fields, Doctoral Dissertation (2008), 16-18.

[17] J. L. Zhang and L. Z. Yang, Some results related to a conjecture of R. Bruck concerning meromorphic functions sharing one small function with their derivatives, Ann. Acad. Sci. Fenn. Math. 32 (2007), no. 1, 141-149.

[18] - A power of a meromorphic function sharing a small function with its derivative, Ann. Acad. Sci. Fenn. Math. 34 (2009), no. 1, 249-260.

FENG L ̈̈

School of Mathematics and Computational Science

China University of Petroleum

Dongying, Shandong, 257061, P. R. China

E-mail address: lvfeng18@gmail.com

HONGXUN YI

Department of Mathematics

SHANDONG UNIVERSITY

Jinan, Shandong, 250100, P. R. China

E-mail address: hxyi@sdu.edu.cn 